\title{
Linear Control Analysis of the Autocatalytic Glycolysis System
}

\author{
Fiona A. Chandra, Gentian Buzi, and John C. Doyle, Member, IEEE
}

\begin{abstract}
Autocatalysis is necessary and ubiquitous in both engineered and biological systems but can aggravate control performance and cause instability. We analyze the properties of autocatalysis in the universal and well studied glycolytic pathway. A simple two-state model incorporating ATP autocatalysis and inhibitory feedback control captures the essential dynamics, including limit cycle oscillations, observed experimentally. System performance is limited by the inherent autocatalytic stoichiometry and higher levels of autocatalysis exacerbate stability and performance. We show that glycolytic oscillations are not merely a "frozen accident" but a result of the intrinsic stability tradeoffs emerging from the autocatalytic mechanism. This model has pedagogical value as well as appearing to be the simplest and most complete illustration yet of Bode's integral formula.
\end{abstract}

\section{INTRODUCTION}

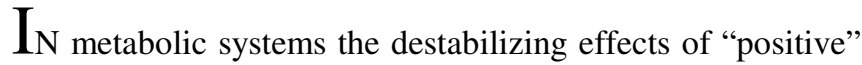
autocatalytic feedback is often countered by negative feedback loops. Instability due to high autocatalysis is typically via a real pole (i.e. saddle-node bifurcation) whereas high inhibition can drive a system into a limit cycle (sustained oscillations via a Hopf bifurcation). This effect has also been studied in other biological systems such as mitogen-activated protein kinase cascades [1].

We wish to explore the hard limits of stability and performance that arise from such autocatalytic and regulatory mechanisms using a familiar and well-understood example. The glycolytic system is ideal to motivate such theoretical analysis for biological systems. Glycolysis is perhaps the most common control system on the planet as it is found in every one of the more than $10^{30}$ cells, from bacteria to human. It has been widely studied and is one of biology's best understood systems. However, despite the extensive experimental and theoretical studies, many questions as to why oscillations occur in glycolysis remain.

Similar to an engineered power plant whose machinery runs on its own energy product, the glycolysis reaction is autocatalytic. Glycolysis generates Adenosine triphosphate

Manuscript received March 12, 2009. This work was supported in part by the Institute for Collaborative Biotechnologies through grant DAAD1903-D-0004 from the U.S. Army Research Office.

F. A. Chandra is with the Department of BioEngineering, California Institute of Technology, Pasadena, CA 91125 USA (phone: 626-395-2313, e-mail: fiona@ caltech.edu).

G. Buzi, is with the Department of Control and Dynamical Systems, California Institute of Technology, Pasadena, CA 91125 USA (e-mail: genti@cds.caltech.edu).

J. C. Doyle is with the Departments of Control and Dynamical Systems and BioEngineering, California Institute of Technology, Pasadena, CA 91125 USA (e-mail: doyle@cds.caltech.edu).
(ATP), which is the cell's energy currency. The ATP molecule contains three phosphate groups and energy is stored in the bonds between these phosphate groups. Two molecules of ATP are consumed in the early steps (hexokinase, phosphofructokinase/PFK) and four ATPs are generated as pyruvate is produced. PFK is also regulated such that it is activated when the Adenosine monophosphate (AMP)/ATP ratio is low; hence it is inhibited by high cellular ATP concentration. This pattern of product inhibition is common in metabolic pathways.

The instability of the glycolytic pathway has been experimentally observed since the 1960s when oscillations of glycolytic intermediates were seen in continuous flow experiments in yeast extracts. Since then, glycolytic oscillations have been studied extensively both experimentally and theoretically. In intact cells, oscillations have been observed in anaerobic conditions. A nice review of the experimental history can be found in [4]. On the theoretical side, the contribution of different enzymes to the period and amplitude of oscillations has been a particular focus [7], [8]. Numerous mathematical models have also been developed. Many of the models attempt to capture in detail the full mechanism of glycolysis, yielding large models that have high fidelity but can obscure fundamental tradeoffs. This in turn has motivated the search for reduced models [2] that highlight the most essential mechanisms.

Early experimental observations suggest that there are two Hopf modes present in the system. Metabolites upstream and downstream of phosphofructokinase (PFK) have $180^{\circ}$ phase difference, suggesting that a two-dimensional model incorporating PFK dynamics might be a reasonable approximation of the system [3]. In this paper we propose a two-state model based on the autocatalytic stoichiometry of ATP and inhibition of ATP (activation by AMP) on PFK.

We will argue that this simple control model captures the most essential dynamics and bifurcations of glycolysis in the specific interplay between autocatalysis and inhibition. This answers one aspect of "why oscillations?" with a simple, sufficient mechanism that is consistent with more complex and detailed models and directly traceable to extensive experimentation. In the context of such simple models, necessary conditions for oscillations in terms of the slope of the function representing the nonlinear effect of ATP on the reaction rate can also be derived [5].

In this paper, we take this modeling and analysis further by considering deeper aspects of "why oscillations?" If this model does indeed capture the essential mechanism, why do real glycolytic pathways have parameter values that allow for 
oscillations? More deeply, why is there this pattern of feedbacks and not some other? (Savageau et al. addresses similar questions in [15]). A number of alternative hypotheses have been put forth. Some suggest that glycolytic oscillations potentially minimize the dissipation of free energy [9], although most argue that they can be detrimental to the cell [10]-[12], and thus it is often claimed that they are merely a "frozen accident" of evolution.

In this paper, we show that glycolytic oscillations are rather a result of the hard tradeoff that emerges from the autocatalytic mechanism of glycolysis, which is necessary for the downstream reactions to proceed. We will further argue that control theory and particularly Bode integral limits provide a clear and coherent framework in which to study and answer these questions, although even deeper analysis will require continued integration of control theory and farfrom-equilibrium thermodynamics [15].

In the control theory community, the idea of performance limitations and trade-offs is well established, most elegantly expressed by the Bode Integral Formula or related inequalities [6]. The Bode formula uses conservation of area under the curve of the Sensitivity function $S$ to show that benefits achieved in certain performance criteria must be paid for in another. In the frequency domain, we typically want $S(j \omega)$ to be small for small steady state error in some frequency range (around $\omega=0$ ). However, pushing this low frequency response to be smaller must be compensated by a larger response in another region. This idea is often visualized as a "water-bed" effect: as we push down on one part of $S$, the displaced "water" inevitably makes another part rise.

This trade-off law is universal in all systems, but may be aggravated in systems with certain structure. Despite its universality, this idea is currently not well-known outside of the control theory community. We ultimately plan to use the glycolysis system to introduce the importance of this tradeoff idea to the wider scientific community, and illustrate the potential of having a more unified theory of hard limits.

The usual Bode Integral Formula assumes degree $\geq 2$. In this paper we state a version that holds as well for systems with degree $<2$ but at least one right half plane (RHP) zero. Proofs of this result are standard and are omitted. We show that our simple glycolysis model is such a system, has severe limits on performance and robustness, is intrinsically prone to oscillations, and that the autocatalysis exacerbates these hard limits. The two-state model we present is the simplest, most complete, and most dramatic example we are aware of that illustrates the tradeoff idea of the Bode formula, and thus should be of widespread pedagogical value. While there is no new control theoretic result per se, we hope this paper will be of interest to control theorists due to its connections with long-standing questions in biology, as well as motivation for more unified theories of hard limits involving energy efficiency in addition to control. Due to space limitations, biological details will unfortunately be somewhat terse.

\section{MATERIALS AND METHODS}

\section{A. Nominal Regulated Autocatalytic Model}

Experimental observations suggest that a two-state model centered on an abstracted version of PFK is a reasonable simplification of glycolysis. Consider a two-state model with $\operatorname{ATP}(x)$ and a lumped intermediate metabolite $(y)$ as states. We assume that the total concentration of adenosine phosphates in the cell remains constant:

$$
[\text { Atot }]=[A T P]+[A D P]+[A M P]
$$

and hence the activating effects of AMP can be expressed as inhibition by ATP. Because we will focus on linearizations, the possible saturating effects of (1.1) will be ignored.

We further assume that the decay rates of the metabolites and the intermediate reaction converting $\mathrm{y}$ to $\mathrm{x}$ are operating in the linear regime and obey simple mass action kinetics:

$$
\begin{aligned}
& \dot{x}=-q \frac{V x^{q}}{1+\gamma x^{h}}+(q+1) k_{y} y-k_{x} \\
& \dot{y}=\frac{V x^{q}}{1+\gamma x^{h}}-k_{y} y
\end{aligned}
$$

The parameter $k_{y}$ represents the lumped metabolic reactions that generate $\mathrm{ATP}$, and $h$ is the gain of the inhibition of the enzymes by ATP. $k_{x}$ represents the ATP demand of the cell, and we assumed that ATP-dependent processes are in saturation, and thus constant (i.e. the control for the glycolytic flux is set by the ATP demand of the cell). As a reminder, glycolytic oscillations have so far mainly been seen in anaerobic conditions, and so there is no additional ATP production from aerobic pathways.

We normalize the model such that the system produces one more molecule of $\mathrm{x}$ than the number consumed (consumes $q x$ molecules and produces $q+1 x$ molecules). We can further non-dimensionalize with respect to the concentration and flux of $x$ such that the steady state concentration of $x$ is $x_{s s}=1$, and nominally $k_{x}=1$. This greatly simplifies the parameterization.

\section{B. Linearization}

The linearization around the non-zero steady state with the above normalization yields:

$$
\left[\begin{array}{c}
\dot{x} \\
\dot{y}
\end{array}\right]=\left[\begin{array}{c}
-q \\
1
\end{array}\right](\sigma(x-1)+1)+\left[\begin{array}{c}
1+q \\
-1
\end{array}\right] k_{y} y+\left[\begin{array}{c}
-1 \\
0
\end{array}\right](1+\delta)(1.3)
$$

\section{GLOSSARY}

$x \quad$ output, ATP leve

$\delta \quad$ input, disturbance in ATP consumption.

$y \quad$ lumped variable of intermediate metabolites downstream of the autocatalytic reaction 


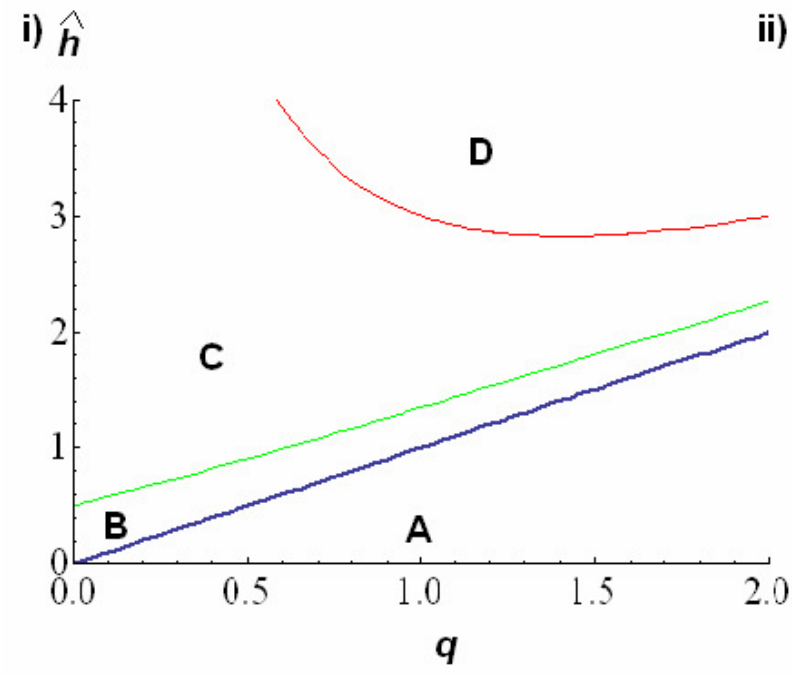

ii) $\mathbf{A}$

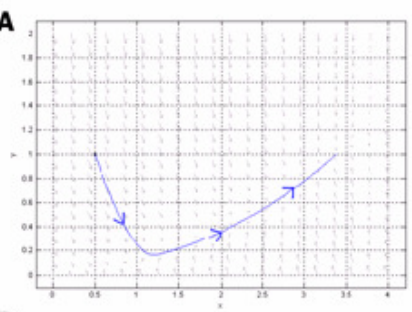

B

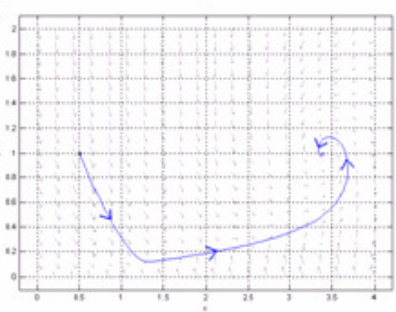

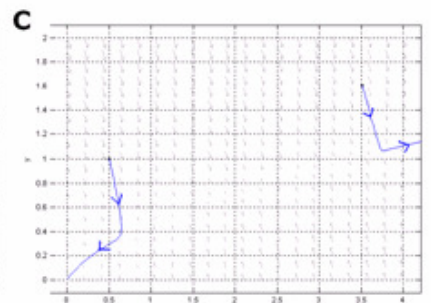

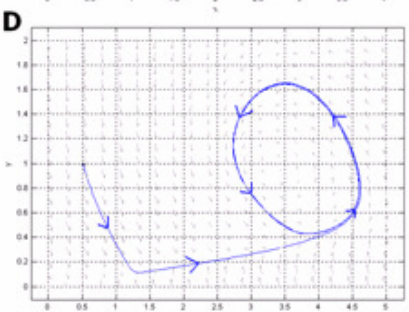

Fig. 1. Stability regions of the system $k_{y}=2$. i) The stability of the system in the $q$ and $\hat{h}$ plane. For a given $q$, there is a range of $\hat{h}$ values where the system is stable. Region A is unstable. Regions C,B are the stable regions, and region D is the oscillatory region. ii) Sample system trajectories in the phase plane domain.

$q$

$\sigma=q-\hat{h} \quad$ linearization of net enzyme (e.g. PFK) response to changes in $x$

$\hat{h}=h\left(1-\frac{1}{V}\right) \quad$ net effective product inhibition gain.

The second term on the right half side is the reaction that consumes $y$ and produces $1+q$ molecules of $x$. The last term is the consumption of $x$, assumed to be saturated, and an external disturbance $\delta$ in consumption is introduced. In glycolysis, 2 ATPs are consumed to produce 4 ATPs at the end. In our model, the reaction is normalized to a consumption of 1 molecule of $x$ to produce 2 molecules of $x$ in the second reaction, which is equivalent to $q=1$. The first term is the reaction involving PFK which consumes $q$ molecules of $x$ and yields one molecule of $y$. Note that $V>$ 1 must hold for a steady state to exist, and small values of $V$ reduce the effective feedback gain. $V$ depends on both the rate and concentration of PFK, and thus larger $V$ requires higher catalytic efficiency, more enzymes, or both.

While there is no separate controller per se, and the terminology of "plant" and controller is perhaps misleading, the sensitivity function $S$ can still be written in the form

$$
S(s)=\frac{P_{h=0}(s)}{P(s)}=\frac{s^{2}+\left(q^{2}+k_{y}\right) s-k_{y} q}{s^{2}+\left(q \sigma+k_{y}\right) s-k_{y} \sigma}
$$

where $P(s)$ can be thought of as the "closed loop" plant from disturbance $\delta$ to output $x$

$$
P(s)=\frac{q s-k_{y}}{s^{2}+\left(q \sigma+k_{y}\right) s-k_{y} \sigma}
$$

and $P_{h=0}(s)$ is simply the "open loop" plant without regulatory feedback, i.e. without inhibition by $\operatorname{ATP}(\hat{h}=0)$.

\section{Theoretical Performance Limits}

If $S(z)=1$ for a right half plane "zero" $z$ (equivalent to a right half plane zero in the open loop plant), which is true for our model, a constraint on the sensitivity function can easily be shown to be:

$$
\frac{1}{\pi} \int_{0}^{\infty} \ln \left|S_{\hat{h}=\hat{h}}(j \omega)\right| \frac{z}{z^{2}+\omega^{2}} d \omega \geq \max \left\{0, \ln \left|\frac{z+p}{z-p}\right|\right\}
$$

where (possibly) $S(p)=0$ (e.g. an OLRHP pole). Note that

$$
\frac{1}{\pi} \int_{0}^{\infty} \frac{z}{z^{2}+\omega^{2}} d \omega=1
$$

so this term constrains the waterbed effect below frequency $z$, a potentially severe limitation.

\section{RESULTS}

\section{A. Dynamics and Stability Region}

Our two-state model seems to capture the essential dynamics of glycolytic oscillations. In continuous stirred tank reactor (CSTR) experiments, oscillations in yeast extracts are seen for a bounded range of flow rates. In a CSTR experiment, materials are flown into and out of the tank at a constant rate such that the volume in the tank remains constant. When the extract and substrate are flown slowly into the reactor, the metabolites do not oscillate. As the flow rate increases, we start to see oscillations and the period increases along with the flow rate until the system settles back into a steady state. This behavior is captured in our model, though this won't be explained in detail here. The flow rates enter as both a constant input into $x$ and $y$ and a "sink" for both states with a constant rate. Phase plane plots show that when this rate is increased, the system goes from a steady state into a limit cycle which expands as the rate 
continues to increase, and eventually returns to a steady state point.

The characteristic polynomial is $s^{2}+\left(q \sigma+k_{y}\right) s-k_{y} \sigma$, so stability holds iff $\min \left\{q \sigma+k_{y},-k_{y} \sigma\right\}>0$, i.e. iff $q<\hat{h}<q+\frac{k_{y}}{q}$. With $q=1, k_{y}=2$, stability only holds for a narrow range of $1<\hat{h}<3$. Local stability regions with respect to the different parameters are shown in Fig. 1. We see that high negative feedback $(\hat{h})$ drives the system into sustained oscillations. However, if there is no autocatalysis $(q=0)$, the system is always stable, and as $q$ is increased, the region of stability becomes narrower.

\section{B. Limits and Tradeoffs: Example of Glycolysis}

Autocatalysis not only narrows the stability region but also exacerbates the system performance, as seen in Figure 2. Our model is simple enough that it can be thoroughly analyzed analytically to explain the intrinsic nature of such oscillations and transients, and the resulting formulae are remarkably simple.

The simplest performance requirement is for small steady state gain $P(0)$ from $\delta$ to $x$. Both $P(0)$ and $S(0)$ are easily computed to be

$$
P(0)=\frac{1}{\sigma}=\frac{1}{q-\hat{h}}, \quad S(0)=\frac{q}{\sigma}
$$

so steady state performance is improved via larger gain $h$, though stability limits this to

$$
|P(0)|>\frac{q}{k_{y}}
$$

At this limit the system would oscillate. Thus large autocatalysis $q$ and small $k_{y}$, which would both be desirable for steady state metabolic efficiency, severely constrains achievable steady state disturbance rejection.

The natural next question is how this would change if PFK control via ATP were allowed to be more complex, without regard to biological implementation. Bode's integral formula suggests that tampering with ATP feedback would not matter. The reason is that the zero of this glycolysis model, given by

$$
z=\frac{k_{y}}{q}
$$

indeed lies in the right half plane, and therefore the performance constraint given above holds for this system. For all $q \geq 0$, there is also one unstable pole in the open loop plant $(\hat{h}=0)$, which is given by:

$$
\begin{aligned}
& p=\text { RHPzero }\left\{s^{2}+\left(q^{2}+k_{y}\right) s-q k_{y}\right\} \\
& =\frac{-q^{2}-k_{y} \pm \sqrt{\left(q^{2}+k_{y}\right)^{2}+4 q k_{y}}}{2}
\end{aligned}
$$

and hence the area under the sensitivity curve obeys the constraint given in equation (1.6). This implies that even if the system is controlled optimally, its performance is limited by the ratio on the right half side of the equation. It is also easy to verify that our biologically motivated model achieves this bound with equality, so could not be uniformly improved on. This is a much stronger result than merely verifying that a given model has performance limitations, because it says that such limitations would apply to all controllers, without regard to their implementation.

The performance limitation of the system is not dependent on $\hat{h}$, but depends only on $q$ and $k_{y}$, since both $z$ and $p$ depend only on these parameters. Further analysis separating the stoichiometry with the exponential coefficient of the autocatalysis shows that $z$ is dependent only on the stoichiometry and not the exponent. This implies that the system performance is limited only by the pathway architecture, in particular the stoichiometry of the autocatalysis. Furthermore, the ratio $\left|\frac{z+p}{z-p}\right|$ from equation (1.6) monotonically increases to infinity as $q$ is made larger and/or $k_{y}$ smaller; therefore the performance limit worsens in exactly the same direction as the steady state gain.

Decreasing $q$ can allow for a better bound, as illustrated in Fig. 2 (generated using MATLAB). The peaks of $S$ increase more significantly as $\hat{h}$ is increased for systems with higher $q$. However, decreasing the stoichiometry will undermine the system's efficiency in generating ATP - yet another tradeoff. Similarly, the tradeoffs are relieved by a larger $k_{y}$, but this requires either more efficient or more abundant enzymes. Full state feedback by adding allosteric control of PFK by the intermediate metabolite y can eliminate the transmission zero but greatly adds to the complexity of the enzyme. Finally, the rest of the cell can contribute to alleviating this tradeoff if consumption is not saturated. The worst case is having saturated consumption, small $k_{y}$, and large $q$. The case of small $k_{y}$ can be achieved when the intermediate glycolytic enzymes are downregulated.

\section{DISCUSSION}

Whereas certain parameters such as the kinetic rates and inhibitory constant of the system might have been tuned by evolution and natural selection, and might naturally be assumed "optimal" in some sense, the autocatalysis and stoichiometry are inherent properties of the pathway's structure. In what sense could these be optimal, when they so aggravate control performance. Our analytical results hold for all $q>0$, regardless of the values of the other parameters. As such, these particular properties of stability and performance limitations are present in the system regardless of the cell's environment and noise and fluctuations, intrinsic or extrinsic.

The plot of the stability region shows that for $q=0$ 
sustained oscillations are not possible in this simple model. Whereas the oscillations occur only when the negative feedback gain is amplified, the autocatalytic structure of the pathway is also necessary. Pomerening et al. have similarly shown experimentally in the case of cell-cycle oscillator that positive feedback is necessary to generate $\mathrm{Cdc} 2$ oscillations [13].

Whenever a system is reduced to a simple model, there is always the question whether or not the results would hold for a more complex, complete model. To address this issue, we have extended our model to: 1) a general $n$-state model, 2) incorporate reversible reactions, and 3) incorporate consumption of intermediate metabolites. We find that our results hold qualitatively even for these extended models (in preparation). Furthermore, we have shown that similar results hold for the nonlinear case (in preparation), although the presentation is substantially more complicated. We have presented only the results for the linearized system in order to keep our model system simple enough to introduce the concept of tradeoffs and performance limitations.

While in the current model we have assumed that the ATP consumption is constant, we have found that changing the consumption to a linear one would affect only $p$ but not $z$.

Whether or not glycolytic oscillations serve a purpose in the cell has been an unsolved debate. Several papers have suggested that glycolysis is more efficient in oscillatory mode than in steady state, based on higher (average) ATP/ADP ratio and lower (average) free-energy dissipation [9], [12]. On the other hand, recent evidence suggests that glycolytic oscillations trigger membrane current oscillations in myocardial cells and result in action potential duration shortening, and may contribute to arrhythmias [10], [14].

Our analysis introduces a new view into this debate on the necessity and the raison d'etre of glycolytic oscillations. The performance hard limits suggest that glycolytic oscillations are not merely a frozen accident that occurred during evolution but a necessary trade-off inherent in the structure of the system. With this level of autocatalysis, glycolysis has inherent performance limitations which will lead to operation near oscillatory regimes.

Whereas the purpose of oscillation per se is unclear, the role of autocatalysis is much better understood. While the details are themselves complex, the standard biochemistry argument (e.g. in [16]) is that steady-state efficiency requires the initial energy investment in the form of phosphate addition, obtained from ATP. Some of the necessary intermediates of glycolysis lie on a higher energy landscape than glucose, thus the energy investment is necessary to reach these intermediate states. Without the addition of these phosphates, the substrate would not be able to proceed through the downstream reactions.

If this argument is accepted, autocatalysis is simply necessary for the steady state efficiencies of glycolysis. The observed oscillations are therefore inevitable consequences of the pathway operating in a high autocatalytic regime $(q=1)$. In addition to steady state efficiency, the system must also be optimized for its regulatory performance, i.e. disturbance rejection, also dominated by disturbances at low frequencies around steady state. Evolution has apparently fine-tuned the system such that it achieves the optimal performance bound and is relatively robust to commonly encountered environmental fluctuations. However, optimizing both steady state efficiency and disturbance rejection gives the system the tendency to oscillate in some circumstances. Therefore, there is no "purpose" per se to the oscillations; they are the side effects of tradeoffs involving efficiency, performance, and robustness.

This is not to say that autocatalysis would automatically lend a system the tendency to oscillate. As seen in our model, the negative feedback is necessary for sustained oscillation. Different autocatalysis and negative feedback interactions can yield different stability and performance properties, such as the threshold 'all-or-none' response seen in the lac operon in E. coli and the blood clotting cascade in humans [17].

The analysis on our simple model of glycolysis illustrates the power of control theory on shedding new light on the cause and necessity of biological phenomenon. The argument above relied on ideas from biochemistry, not described in any detail, to explain the need for autocatalysis and then control theory for the consequences. This clearly motivates a theory that would more explicitly and rigorously treat the tradeoff between steady state efficiency, steady state disturbance rejection, and Bode-type integrals. Currently efforts are being made in unifying thermodynamics and control theory. Although progress has been made in this area, it does not yet address the complexity of reactions such as the ones seen in glycolysis [18].

\section{ACKNOWLEDGMENT}

The authors thank H. Schmidt for his ODE model file.

\section{REFERENCES}

[1] B. Kholodenko, "Negative feedback and ultrasensitivity can bring about oscillations in the mitogen-activated protein kinase cascades." Eur J Biochem vol. 267, pp. 1583-1588, Mar 2000.

[2] F. Hynne, S, Danø, P. O. Sørensen, "Full-scale model of glycolysis in Saccharomyces cerevisiae." Biophys Chem. vol. 94, no. 1-2, pp. 12163, Dec 2001.

[3] A. Betz, B. Chance, "Phase relationship of glycolytic intermediates in yeast cells with oscillatory metabolic control." Arch Biochem Biophys. vol. 109, pp. 585-594, Mar 1965.

[4] P. Richard, "The rhythm of yeast." FEMS Microb Rev, vol. 27, pp. 547-557, Oct 2003.

[5] J. Coatleven, C. Altafini. "A kinetic mechanism inducing oscillations in simple chemical reactions networks." in Proc. of the 47th IEEE Conf. on Decision and Control, Cancun, Mexico, December 2008.

[6] K. Astrom, R. M. Murray, Feedback Systems: An Introduction for Scientists and Engineers. Princeton: Princeton University Press, 2008.

[7] B. Teusink, B. M. Bakker, H. V. Westerhoff, "Control of frequency and amplitudes is shared by all enzymes in three models for yeast glycolytic oscillations." Biochim. Biophys. Acta vol. 1275 no. 3, pp. 204-12, Jul 1996. 

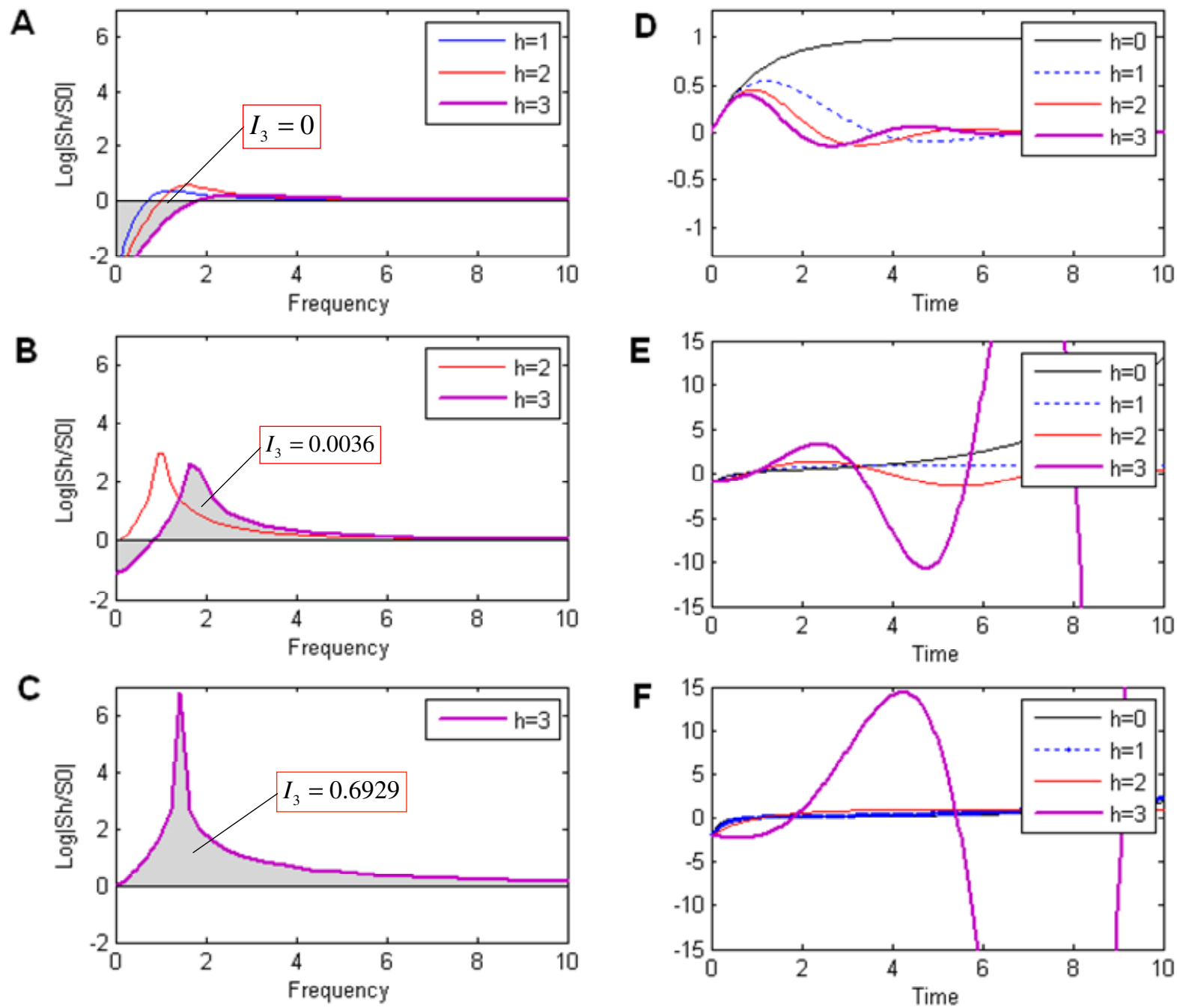

Fig. 2. Sensitivity functions and time trajectories of the 2-state glycolysis system with $k_{y}=2$ for different feedback gain $h$. A, B, C, Sensitivity functions for the system with autocatalysis $q=0,1,2$, respectively. $h$ in the figure legends corresponds to the effective feedback gain $\hat{h}$. When the system is in the stable region, as $\hat{h}$ is increased, the peak of the curve increases. Frequency plots for $\hat{h}$ such that the system lies in the unstable region (Region A, Fig. 1) are not shown. D, E, F, Time trajectories of the same systems demonstrate markedly increased transient oscillations, and for $q>0$ at high enough $\hat{h}$, the system goes into sustained oscillations. The integral for $\hat{h}=3$ is given for each case.

[8] M. Bier, B. Teusink, B. N. Kholodenko, H. V. Westerhoff, "Control analysis of glycolytic oscillations." Biophys Chem. vol. 62, no.1-3, pp. 15-24, Nov 1996.

[9] P. E. Richter, J. Ross, "Concentration oscillations and efficiency: glycolysis." Science vol. 211, no. 4483, pp. 715-717, Feb 1981.

[10] B. O'Rourke, B. Ramza, E. Marban, "Oscillations of membrane current and excitability driven by metabolic oscillations in heart cells." Science vol. 265, no. 5174, pp. 962-966, Aug 1994.

[11] J. R. Pomerening, S. Y. Kim, J. E. Ferrell, Jr, "Systems-Level Dissection of the Cell-Cycle Oscillator: Bypassing Positive Feedback Produces Damped Oscillations." Cell vol. 122, no. 4, pp. 565-578, Aug 2005.

[12] Y. Termonia, J. Ross J, "Oscillations and control features in glycolysis: numerical analysis of a comprehensive model." PNAS vol. 78, no. 5, pp.2952-6, May 1981.

[13] R. Frenkel, "Reduced diphosphopyridine nucleotide oscillations in cell-free extracts from beef heart." Arch Biochem Biophys vol. 115, pp.112-21, Jul 1966.
[14] D. N. Romashko, E. Marban, B. O'Rourke, "Subcellular metabolic transients and mitochondrial redox waves in heart cells." PNAS vol. 95, pp. 1618-23, Feb 1998.

[15] R. Alves, M. A. Savageau, "Extending the Method of Mathematically Controlled Comparison to Include Numerical Comparisons." Bioinformatics vol. 16, pp. 786-98, Sep 2006.

[16] D. Voet, J. G. Voet, Biochemistry 3rd Edition. New York: John Wiley \& Sons, Inc, 2004.

[17] T. A. Carrier, J. D. Keasling, "Investigating Autocatalytic Gene Expression Systems through Mechanistic Modeling." J. theor. Biol. vol. 201, pp. 25-36, Dec 1999.

[18] H. Sandberg, J. C. Delvenne, J. C. Doyle, "Linear-Quadratic-Gaussian Heat Engines." in Proc. of the 46th IEEE Conf. on Decision and Control, New Orleans, Dec 2007. 